\section{ONLINE: A general-purpose BASIC microcomputer program to control and record events for operant conditioning experiments}

\section{MARK LEBERER}

Fayetteville State University, Fayetteville, North Carolina 28301

This paper describes a general-purpose on-line microcomputer program to control and record events for a variety of simple reinforcement schedules in up to six operant chambers simultaneously. Since the program is written in BASIC, it should have wide applicability regardless of the computer manufacturer.

Description. ONLINE is a compiled BASIC language program designed to service from one to six operant chambers, each running under an independent simple reinforcement schedule. The program works by sampling a parallel input port at a rate of 100 times/sec and testing the input ports' bit pattern against a stored table of bit patterns. Events are flagged when any prescheduled bit of an input port (for example, reflecting a response in an operant chamber) is "on." The program then measures the event duration in $.01-\mathrm{sec}$ units and increments the response counter by one. When the event ends, the program tests the interresponse time (IRT) counter and/or the response counter to determine if reinforcement delivery is scheduled. If reinforcement is scheduled, the appropriate bit on the output port is turned on for the scheduled reinforcement duration. Following either the end of each response or the end of each reinforcement, the program tests to see if the accumulated session time or reinforcement delivery count limits have been reached. If so, the session for that operant chamber is terminated by turning off the prevailing stimulus conditions bit on the parallel output port. In addition, no further events for that operant chamber are recorded. When the session criteria for all operant chambers are met, the program displays the elapsed session time, the total number of responses emitted, and the number of reinforcements delivered to each operant chamber. The operator is then given the option of storing on disk or tape the summary data, as well as the response duration and IRT distributions for each operant chamber. Control then returns to the resident disk operating system.

Hardware Requirements. The system was developed on a SWTP $6800^{1}$ microcomputer using ABASIC, a BASIC language compiler supplied by Microware Systems Corporation. ${ }^{2}$ The development system had $32 \mathrm{~K}$ of RAM, with dual-floppy disks, a single 8-bit parallel input/output (I/O) port, and an interrupt timer, all supplied by SWTP.

By modifying the size of the values in the dimension statement, the program can be made to occupy less memory or to accommodate a greater number of operant chambers. In addition, since all $\mathrm{I} / \mathrm{O}$ occurs in subroutines, the program can easily be modified or adapted to other machine manufactures with unique I/O requirements.

In addition, simple modification of the print statements following Line 9000 (see program listing in the Appendix) permits the user with no disk system to display the frequency distributions of IRT and response duration on a printing terminal or cassette tape.

Availability. Requests for additional information or for a copy of the BASIC source code and the compiled machine language code on SWTP diskette should be addressed to the author in care of the Psychology Department, Fayetteville State University, Fayetteville, North Carolina 28301. (If a copy of the program on SWTP diskette is desired, please send a check, payable to the author, for $\$ 15$ to cover the cost of the diskette and postage.)

\section{NOTES}

1. Southwest Technical Products Corporation, 219 West Rhapsody, San Antonio, Texas 78216.

2. Microware Systems Corporation, 5835 Grand Avenue, P.O. Box 4865, Des Moines, lowa 50304.

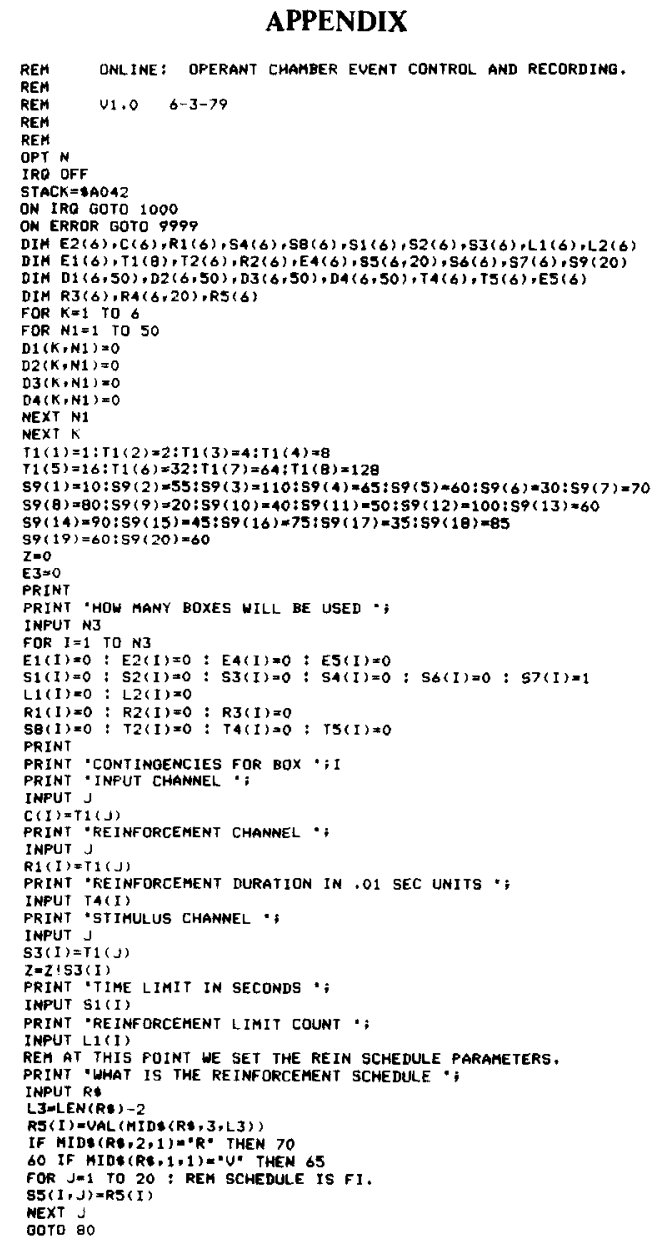



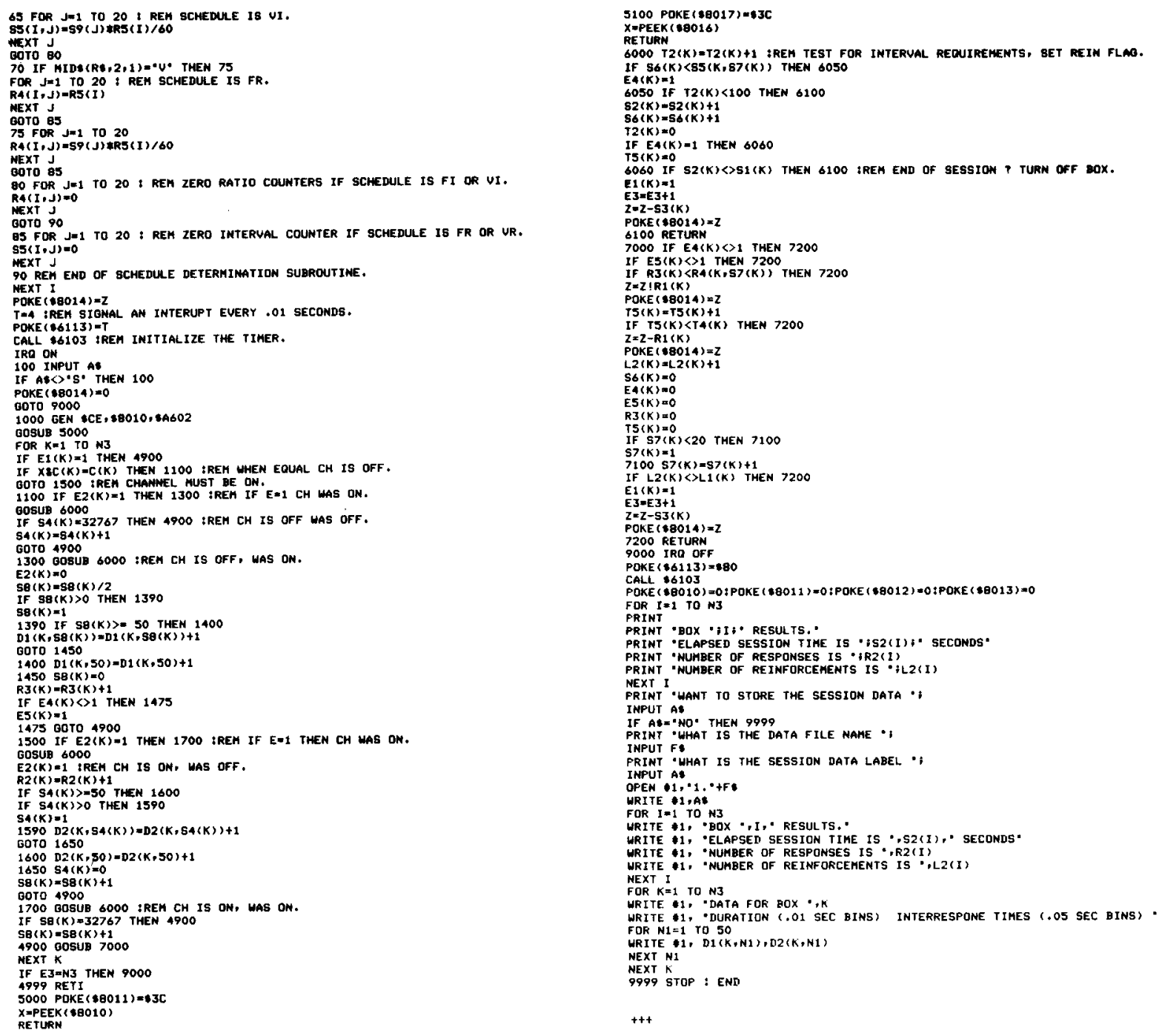

(Accepted for publication March 25, 1980.) 